\title{
VALIDEZ DIAGNÓSTICA DE LA ESCALA DE ALVARADO EN PACIENTES CON DOLOR ABDOMINAL SUGESTIVO DE APENDICITIS, EN UN HOSPITAL DE TERCER NIVEL, DEL SUR DE BOGOTÁ
}

\section{DIAGNOSTIC VALIDITY OF THE ALVARADO SCALE IN PATIENTS WITH APPENDICITIS, SUGESTIVE OF ABDOMINAL PAIN, IN A THIRD LEVEL HOSPITAL OF SOUTHERN BOGOTA}

\author{
Karen Viviana Peralta R. ${ }^{1}$, Carlos Andrés Caballero R. ${ }^{2}$, Mónica del Pilar Mora V. ${ }^{3}$
}

\begin{abstract}
${ }^{1}$ Estudiante Programa de Medicina XII semestre. Universidad de Ciencias Aplicadas y Ambientales U.D.C.A, Calle 108 N$^{\circ}{ }^{a} 29$, Bogotá, D.C, e-mail: nanita_7691@hotmail.com; ${ }^{2}$ Estudiante programa de Medicina XII semestre. Universidad de Ciencias Aplicadas y Ambientales U.D.C.A, Carrera 71b № 74 87, e-mail: cc92dr@hotmail.com; ${ }^{3}$ Médico, Especialista en Cirugía General, Cirujana general Subred Integrada de Servicios de Salud Sur, USS Tunal momi_kita@hotmail.com
\end{abstract}

Rev. U.D.C.A Act. \& Div. Cient. 20(1): 5-11, Enero Junio, 2017

\section{RESUMEN}

El dolor abdominal agudo es uno de los síntomas por los que un paciente asiste al servicio de urgencias de cualquier hospital, a nivel mundial, representando entre un 5 y $10 \%$ del total de consultas, constituyéndose para el médico de urgencias y especialistas interconsultados, un reto, dado que encierra varias patologías, que pueden ser o no quirúrgicas y que de no ser diagnosticadas, correcta y oportunamente, se pueden convertir en fatales, como la apendicitis, la condición patológica y quirúrgica, la que, frecuentemente, se ve enfrentado el cirujano general. El paciente, se puede presentar con signos y síntomas muy variados, que van desde un cuadro claro de apendicitis o no tener signos ni síntomas que sugieran, como diagnóstico, esta patología, por lo cual, se han desarrollado escalas que faciliten o aporten ayuda al médico para un correcto diagnóstico y posterior tratamiento. La escala de Alvarado hace parte de estas ayudas diagnósticas, siendo un método económico, no invasivo, rápido y confiable. Se evaluó por medio de este proyecto, la validez de la escala en la USS Tunal de IIII nivel, al ser uno de los hospitales más grandes y concurridos de Bogotá; para ello, se tomó una muestra de 161 pacientes y valorando cada parámetro de la escala, además, de comparar el puntaje dado por la misma y el diagnóstico histopatológico. Los resultados permiten concluir que la escala es sensible, pero no específica para el diagnóstico de apendicitis.

\section{SUMMARY}

Acute abdominal pain is one of the symptoms which a patient attends the emergency room of any hospital worldwide, representing a 5 to $10 \%$ of those consultations; this represents for the emergency doctor and specialists interconsulted a challenge, because it contains several conditions that may or may not be surgical and not be diagnosed properly and promptly can be fatal, within these, is appendicitis, pathological and surgical condition which most often is faced the surgeon general. The patient may present with signs and varied symptoms ranging from a clear picture of appendicitis, or have no signs or symptoms suggestive as this pathology diagnosis; so scales have been developed to facilitate or provide help to the doctor for a correct diagnosis and subsequent treatment; Alvarado makes the scale of these diagnostic aids, being a non-invasive, fast and reliable economical method. Was evaluated by means of this project the validity of the scale in the USS Tunal IIII level to be one of the largest and busiest hospitals in Bogota, for this sample of 161 patients was taken Hospital and rated each parameter in addition to comparing the scores given by the same scale and histopathological diagnosis. The results show that the scale is sensitive but not specific for the diagnosis of appendicitis.

Key words: Appendicitis, Alvarado scale, diagnosis, false positives.

Palabras clave: Apendicitis, escala de Alvarado, diagnóstico, falsos positivos. 


\section{INTRODUCCIÓN}

La apendicitis es la urgencia abdominal que mayormente se presenta a lo largo del mundo. La literatura reporta que un $7 \%$ de la población mundial presentará apendicitis en su vida, de acuerdo al reporte de Ospina et al. (2011) y su importancia radica en que es una de las patologías más consultadas en los servicios de urgencias y, al no ser diagnosticada oportunamente, puede ser mortal.

Los signos y los síntomas predictivos de esta patología son dolor en cuadrante inferior derecho, defensa abdominal y dolor abdominal migratorio, desde la región periumbilical al cuadrante inferior derecho (Paulson et al. 2003). Su diagnóstico es primordialmente clínico, aunque las variaciones en la posición del apéndice y su relación con otras estructuras intra abdominales la hacen un reto para el médico de atención inicial y para el cirujano general, quien, finalmente, determina si el cuadro clínico es quirúrgico o no. Anatomopatológicamente esta entidad consta de cuatro fases: congestiva o edematosa, supurativa o fibrinopurulenta, gangrenosa y perforada (Caycedo, 2013).

Con el fin de disminuir la morbimortalidad se han introducido, a lo largo de la historia, varias escalas, que buscan identificar el cuadro de apendicitis aguda complicada de la no complicada, o de discriminar el nivel de riesgo en el que se encuentra el paciente. Uno de estos es el sistema de Alvarado, un test que se basa en la historia referida por el paciente, los hallazgos al examen físico y algunos exámenes de laboratorio (Singh et al. 2008) que, al sumarlos, generan un puntaje que indica el riesgo que tiene el paciente de estar padeciendo o no apendicitis y el manejo a seguir, de acuerdo a la puntuación. Fue desarrollado por el médico colombiano Alfredo Alvarado (De Quesada et al. 2015), quien en 1986, al realizar un estudio retrospectivo con 305 pacientes apendicectomizados, identificó ocho factores predictivos para el diagnóstico clínico de apendicitis, como se puede observar en la tabla 1: tres síntomas, cada uno con un puntaje de 1 : dolor abdominal migratorio a fosa ilíaca derecha, anorexia, náuseas/vómito; tres signos: signo de blumberg presente, es decir, rebote positivo o dolor a la descompresión de la fosa ilíaca derecha y fiebre $>37,5^{\circ} \mathrm{C}$, ambas con un puntaje de 1 y dolor en cuadrante inferior derecho, variable con 2 puntos y, por último, dos exámenes de laboratorio: leucocitosis $>10.500 \mathrm{cel} / \mathrm{mm}^{3}$, con un puntaje de 2 y neutrofilia $>75 \%$ (Hernández et al. 2012), siendo el puntaje máximo 10.

De este modo, la sumatoria de las variables presentes en cada paciente permite ubicarlo en un riesgo bajo, con un $33 \%$, de padecer apendicitis en el momento de la valoración; riesgo intermedio, con un $66 \%$, de padecer apendicitis en el momento de la valoración o riesgo alto, con un $93 \%$, de padecer apendicitis en el momento de la valoración y, a la vez, sugiere la conducta a seguir con ese paciente, como indica Sanabria et al. (2010): riesgo bajo (0 a 4 puntos): egreso temprano y control ambulatorio a las 24 horas; riesgo intermedio (5 a 7 puntos): tomografía computarizada (TC) abdominal temprana y decisión, según los hallazgos (laparotomía para los casos de TC positiva y egreso, con control ambulatorio a las 24 horas, para los pacientes con TC negativa) y riesgo alto (8 a 10 puntos): cirugía inmediata (Ohle et al. 2011).

Este test tiene la ventaja de haber sido sujeto a varios estudios de validación, los que confirman que es un método simple, fácil y barato, orientado al diagnóstico clínico de la apendicitis y que no conlleva de mayor esfuerzo del médico, ya sea médico general o cirujano experimentado (Khan \& Ur, 2005; Ricci et al. 2015).

Tabla 1. Escala de Alvarado (Ohle et al. 2011).

\begin{tabular}{|l|c|}
\hline Parámetro & Puntaje \\
\hline Dolor abdominal migratorio & 1 \\
\hline Anorexia & 1 \\
\hline Náuseas/vómito. & 1 \\
\hline Dolor en cuadrante inferior derecho & 2 \\
\hline Fiebre (Temperatura $>37,5^{\circ} \mathrm{C}$ ) & 1 \\
\hline Rebote positivo (blumberg presente) & 1 \\
\hline Leucocitosis (leucocitos $>10.500 \mathrm{cel} / \mathrm{mm}^{3}$ ) & 2 \\
\hline Desviación a la izquierda (neutrofilia $>75 \%)$ & 1 \\
\hline Total & 10 \\
\hline
\end{tabular}

\section{MATERIALES Y MÉTODOS}

Componente ético: Según la Resolución 8430 (Ministerio de Salud, 1993), este proyecto trata de una investigación sin riesgo, en la que se recogerán datos, mediante la revisión de historias clínicas y resultados obtenidos de la aplicación de una encuesta cerrada, diseñada para el estudio, que evalúa la presencia o no de las variables propuestas para el mismo, de modo que no se interviene directamente con "las variables biológicas, fisiológicas, sicológicas o sociales de los individuos que participan en el estudio"; se considera no hay riesgo para la integridad de los pacientes, por lo que no se solicita consentimiento informado.

El proyecto fue revisado y aprobado por el comité de ética para la investigación de la USS Tunal. Se diseñó un estudio observacional de corte transversal para evaluar la validez de la escala de Alvarado durante junio y diciembre de 2015, en pacientes de 18 a 50 años de edad. Se comparó el puntaje obtenido, luego del examen médico, aplicando esta escala con el diagnóstico histopatológico definitivo, en los pacientes a los que se les extirpó el apéndice. Para ello, se creó 
un instrumento que incluyó las ocho variables propuestas por la escala de Alvarado y el sexo del paciente, el puntaje obtenido (0 a 4; 5 a 7; 8 a 10), el hallazgo quirúrgico, el informe histopatológico y la opción de diagnóstico definitivo, en caso de que el diagnóstico histopatológico fuera negativo para apendicitis.

Se incluyeron los pacientes de 18 a 50 años de edad que consultaron por dolor abdominal en fosa ilíaca derecha, que fueron interconsultados al servicio de cirugía general de la USS Tunal, durante el periodo evaluado. Se excluyeron los pacientes fuera del rango etario mencionado, con apendicectomía previa, pacientes con dolor abdominal no localizado en fosa ilíaca derecha y pacientes femeninas en estado de gestación.

Teniendo en cuenta las estadísticas del DANE, en 2015 la población total de Bogotá fue 7'878.783 personas (DANE Proyecciones de población total. Periodo 2005-2020) y, de acuerdo a la literatura, el $7 \%$ de la población, en general, padecerá apendicitis aguda, en algún momento de su vida (Ospina et al. 2011), lo cual, quiere decir que es probable que, durante el 2015, 551.514 personas residentes en Bogotá podrían haber padecido apendicitis, es decir, 275.759 personas, por semestre. Por lo que se tomó una muestra representativa de 161 casos de dolor sugestivo de apendicitis, que se presentaron durante el periodo de junio a diciembre de 2015 y que fueron interconsultados al servicio de cirugía general de la USS Tunal, para estudiar la validez de la escala de Alvarado. Cabe mencionar que la población total con dolor abdominal localizado en fosa ilíaca derecha, sin discriminar pacientes, fue de 9.644 .

Se solicitó en el área de sistemas del hospital la base de datos de pacientes que consultaron al servicio de urgencias por dolor abdominal sugestivo de apendicitis y posteriormente fueron remitidos al servicio de cirugía general de este hospital para su valoración, realizada en un comienzo por el estudiante o interno del servicio y ratificada después por el residente o cirujano general de turno; se tomó una muestra aleatoria de 161 casos, correspondiente a la población finita, que se mencionó anteriormente, es decir, 275.759 personas y durante tres días se revisaron las historias clínicas correspondientes.

Los datos recogidos se analizaron Mediante comandos en Stata 10 ® y MedCalc Statistical Software versión 17.4, y Excel 2013. Los dos primeros, se usaron para determinar los puntos de corte y la curva ROC y, el segundo, permitió hallar las frecuencias y el promedio de los datos.

\section{RESULTADOS Y DISCUSIÓN}

El dolor abdominal es uno de los motivos de consulta más frecuentes al servicio de urgencias (Bejarano et al. 2011), siendo la apendicitis una de las entidades más usuales dentro de sus causas, convirtiéndola en un motivo constante de interconsulta al servicio de cirugía general, donde se revalora al paciente, teniendo en cuenta ciertos parámetros, que permiten una sospecha bastante cercana al diagnóstico de apendicitis aguda. Estos parámetros (defensa abdominal en fosa ilíaca derecha, rebote positivo-blumberg presente, temperatura $>37,5^{\circ} \mathrm{C}$, náuseas o vómito, dolor abdominal migratorio, anorexia, leucocitosis $>10.500 / \mathrm{mm}^{3}$ y neutrofilia $>75 \%$ (Ohle et al. 2011), forman parte de la escala de Alvarado, que ha sido usada para clasificar a los pacientes en tres tipos de riesgo: bajo, intermedio y alto; esto determinará el manejo que le será dado a cada uno. Los parámetros evaluados son: defensa abdominal en fosa ilíaca derecha, rebote positivo (blumberg presente), temperatura $>37,5^{\circ} \mathrm{C}$, náuseas o vómito, dolor abdominal migratorio, anorexia, leucocitosis $>10.500 / \mathrm{mm}^{3}$ y neutrofilia $>75 \%$ (Ohle et al. 2011).

El diagnóstico de apendicitis es netamente clínico y aunque no haya un signo que con certeza prediga la presencia de la enfermedad, el puntaje dado por la escala de Alvarado permite una aproximación al diagnóstico, reduciendo, así, la extirpación de apéndices sanos (Ricci et al. 2015). Es por ello, que en la guía de práctica clínica para apendicitis de la USS Tunal, la escala de Alvarado se encuentra contemplada para el diagnóstico de la misma (Vega et al. 2013).

Durante la recolección de los datos para este estudio, se logró identificar que se acata en las distintas áreas la guía de práctica clínica para aproximarse al diagnóstico clínico de apendicitis aguda, puesto que se evalúa al paciente buscando los parámetros enunciados, en la escala mencionada, permitiendo la clasificación, para posterior intervención, sea quirúrgica o no. El promedio de edad fue 30,5 años y de las 161 historias clínicas estudiadas, el 57\% correspondió al género femenino y el $43 \%$, al masculino.

Al haber hecho la clasificación de riesgo, según el puntaje obtenido tras la evaluación, se obtuvo que el riesgo de mayor frecuencia fue el intermedio, $42 \%$ de los casos (Tabla 2), determinando la conducta a seguir. Cabe aclarar que, aunque la literatura expone un manejo conservador en los casos de bajo riesgo, en el $22 \%$ de los pacientes se usó ecografía, beneficiándose un $19 \%$, del $22 \%$ (86\%), pues confirmó el diagnóstico de apendicitis aguda. El mayor uso de métodos complementarios imagenológicos (ecografía/TAC) fue aplicado al grupo de riesgo intermedio, con un $75 \%$, tal como 
se indica en la bibliografía revisada (Sanabria et al. 2010). En el grupo de alto riesgo, $3 \%$ de los pacientes requirió uso de TAC, lo cual, permitió descartar el diagnóstico de apendicitis aguda. Es necesario mencionar que el $13 \%$ de los pacientes que fueron identificados como riesgo intermedio, por la persistencia y la gravedad de la sintomatología, fueron llevados inmediatamente a cirugía, sin esperar la ayuda imagenológica.

Tabla 2. Riesgo encontrado en el estudio según escala de Alvarado.

\begin{tabular}{|l|c|}
\hline Riesgo & Porcentaje \\
\hline Alto & 39 \\
\hline Bajo & 19 \\
\hline Intermedio & $\mathbf{4 2}$ \\
\hline Total & $\mathbf{1 0 0}$ \\
\hline
\end{tabular}

El diagnóstico de apendicitis histopatológicamente se confirmó en 103 casos del total de la muestra, siendo más frecuente en el sexo masculino, con un 52\% (Ospina et al. 2011; Alarcón, 2012). Los 58 casos restantes, se dividieron de la siguiente manera: $22 \%$, no requirió cirugía, resolviendo el cuadro con manejo médico; 9.9\%, se intervino quirúrgicamente, hallando otra patología y, 3,1\%, solicitó salida voluntaria (Cuadro 1).
Del 9,9\% que correspondió al diagnóstico histopatológico o quirúrgico negativo para apendicitis, el $73 \%$ (11 pacientes) fueron mujeres, coincidiendo con lo descrito por Sanei et al. (2009), Rossini et al. (2009), Velázquez et al. (2010) y Ohle et al. (2011), al evaluar la escala de Alvarado en pacientes femeninas; en edad fértil, la patología ginecológica suele ser enmascarada por los signos sugestivos de apendicitis.

En cuanto a los parámetros a evaluar en la escala de Alvarado, el síntoma más frecuente fue náuseas/vómito, con un 81\%; el signo que más se encontró fue defensa abdominal, al valorar la fosa ilíaca derecha, con un $67 \%$; este hallazgo coincide con el estudio adelantando por Hernández et al. (2012) y el paraclínico que, de manera habitual, se encontró positivo, fue leucocitosis, con un $77 \%$ (Tabla 3 ).

La validez de la escala de Alvarado en la USS Tunal, se evaluó mediante la sensibilidad y la especificidad, que tienen como función juzgar la prueba como tal y mediante el valor predictivo positivo y el valor predictivo negativo, que determinan la capacidad que tiene la prueba de diagnosticar o descartar la presencia de la enfermedad.

En la tabla 4, se presentan las características operativas de la escala de Alvarado, de acuerdo a cada punto de corte. El área bajo la curva (ROC) fue de 0,85 (gráfica 1), la sensibilidad obtenida es $68,9 \%$ y la especificidad es $86,2 \%$; lo hallado

Cuadro 1. Diagnósticos negativos para apendicitis.

\begin{tabular}{|l|l|l|}
\hline \multicolumn{1}{|c|}{ Riesgo } & No requirió intervención quirúrgica & Requirió intervención quirúrgica \\
\hline \multirow{3}{*}{ Bajo } & $\begin{array}{l}\text { Dolor abdominal resuelto } \\
\text { Impactación fecal } \\
\text { IVU } \\
\text { Menstruación retrograda } \\
\text { Prostatitis aguda } \\
\text { Salida voluntaria } \\
\text { Salpingitis y ooforitis derechos } \\
\text { Vaginitis }\end{array}$ & Vólvulo \\
\hline \multirow{5}{*}{ Intermedio } & $\begin{array}{l}\text { Dolor abdominal resuelto } \\
\text { IVU } \\
\text { Menstruación retrograda } \\
\text { Quiste de vario } \\
\text { Salida Voluntaria } \\
\text { Vulvovaginitis }\end{array}$ & $\begin{array}{l}\text { Absceso tubárico derecho } \\
\text { Dolor abdominal resuelto } \\
\text { Embarazo ectópico } \\
\text { Hemoperitoneo }\end{array}$ \\
\hline \multirow{2}{*}{ Alto } & Isquemia mesentérica \\
& Quiste ovárico derecho & Plastrón apendicular \\
\hline & & $\begin{array}{l}\text { Ulcera péptica perforada } \\
\text { Piosalpinx bilateral } \\
\text { Salpingitis y ooforitis }\end{array}$ \\
\hline
\end{tabular}


Tabla 3. Distribución de las variables en la población estudiada.

\begin{tabular}{|l|c|}
\hline \multicolumn{1}{|c|}{ Variable } & Porcentaje \\
\hline Náuseas o vómito & $81 \%$ \\
\hline Leucocitosis $>10.500 / \mathrm{mm}^{3}$ & $77 \%$ \\
\hline Anorexia & $73 \%$ \\
\hline Defensa abdominal, fosa ilíaca derecha & $67 \%$ \\
\hline Neutrofilia $>75 \%$ & $66 \%$ \\
\hline Signo de Rebote positivo. Signo de Blumberg positivo & $63 \%$ \\
\hline Dolor abdominal migratorio & $58 \%$ \\
\hline Temperatura (fiebre) $>37.5^{\circ} \mathrm{C}$ & $27 \%$ \\
\hline
\end{tabular}

coincide con los resultados de otros estudios revisados (Andersson \& Andersson, 2008; Cedillo et al. 2012; Sanabria et al. 2010).
Se concluye, en este estudio, que la escala de Alvarado es aplicada, de manera adecuada, a los pacientes que son interconsultados al servicio de cirugía general de la USS Tunal,

Tabla 4. Características operativas por puntos de corte de la escala de Alvarado.

\begin{tabular}{|c|c|c|c|c|c|c|}
\hline Punto de corte & Sensibilidad & $\mathbf{9 5 \%}$ CI & Especificidad & $\mathbf{9 5 \% ~ C I}$ & LR+ & LR- \\
\hline$\geq 1$ & 100,00 & $96,5-100,0$ & 0,00 & $0,0-6,2$ & 1,00 & \\
\hline$>2$ & 100,00 & $96,5-100,0$ & 15,52 & $7,3-27,4$ & 1,18 & 0,00 \\
\hline$>3$ & 98,06 & $93,2-99,8$ & 27,59 & $16,7-40,9$ & 1,35 & 0,070 \\
\hline$>4$ & 96,12 & $90,4-98,9$ & 46,55 & $33,3-60,1$ & 1,80 & 0,083 \\
\hline$>5$ & 86,41 & $78,2-92,4$ & 67,24 & $53,7-79,0$ & 2,64 & 0,20 \\
\hline$>6$ & 68,93 & $59,1-77,7$ & 86,21 & $74,6-93,9$ & 5,00 & 0,36 \\
\hline$>7$ & 55,34 & $45,2-65,1$ & 91,38 & $81,0-97,1$ & 6,42 & 0,49 \\
\hline$>8$ & 29,13 & $20,6-38,9$ & 98,28 & $90,8-100,0$ & 16,89 & 0,72 \\
\hline$>9$ & 5,83 & $2,2-12,2$ & 100,00 & $93,8-100,0$ & & 0,94 \\
\hline$>10$ & 0,00 & $0,0-3,5$ & 100,00 & $93,8-100,0$ & & 1,00 \\
\hline
\end{tabular}

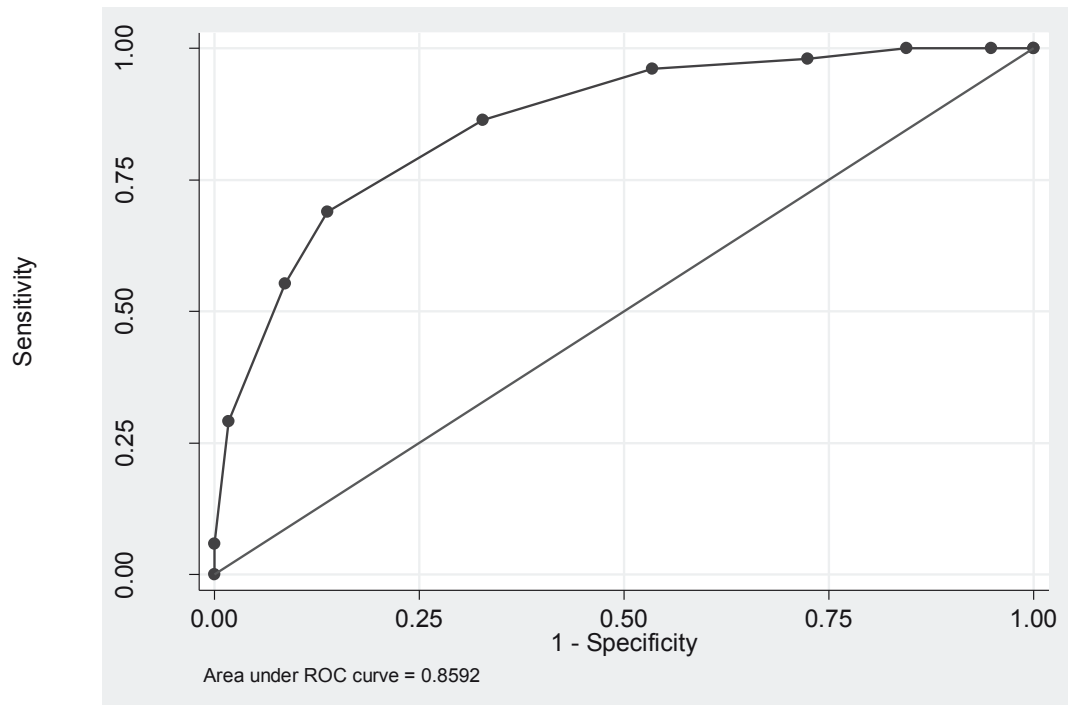

Gráfica 1. Curva ROC de la escala de Alvarado. 
por dolor abdominal sugestivo de apendicitis aguda; se hace un buen uso de la misma, lo que permitió, en la mayoría de los casos, una relación positiva del puntaje dado por la escala, con el reporte histopatológico para apendicitis. A pesar de esto, es claro que la misma es susceptible de presentar falsos positivos, los cuales, son más frecuentes en el riesgo intermedio y en el género femenino. La escala de Alvarado es de gran utilidad, como prueba diagnóstica.

Agradecimientos. A la unidad de servicios de salud (USS) Tunal por hacer posible la realización de este estudio, con base en la población que allí acude; de manera especial, a la Dra. Mónica Mora, especialista en cirugía general y cirujana de la USS Tunal, cargo que, como asesora, hizo que este proyecto tuviera el enfoque correcto; al Dr. Néstor Suárez, epidemiólogo de la USS Tunal y coordinador de docencia servicio, por su ayuda, en cuanto al análisis de los resultados del estudio y a la señora Mercedes Rincón, por su colaboración en la realización de este proyecto. Financiación: El presente trabajo fue financiado por los autores. Conflicto de intereses: El manuscrito fue preparado y revisado por los autores, quienes declaramos que no existe conflicto de intereses que ponga en riesgo la validez de los resultados presentados.

\section{BIBLIOGRAFÍA}

1. ALARCÓN, N. 2012. Asociación entre escala de Alvarado y diagnóstico de apendicitis aguda complicada y no complicada según anatomía patológica en el Centro Médico Naval. Rev. Horizonte Médico. 12(2):14-20.

2. ANDERSSON, M.; ANDERSSON, R. 2008. The apendicitis inflammatory response score: A tool for the diagnosis of acute apendicitis that out performs the Alvarado score. World J. Surg. 32:1843-1849.

3. BEJARANO, M.; GALLEGO, C.; GÓMEZ, J. 2011. Frecuencia de abdomen agudo quirúrgico en pacientes que consultan al servicio de urgencias. Rev. Col. Cir. 26(1):33-41.

4. CAYCEDO, R. 2013. Abdomen agudo. En: Caycedo, R. (ed). Médica Celsus, Cirugía general, 3rd ed. Colombia: Celsus. Bogotá, Colombia. 303p.

5. CEDILLO, E.; SANTANA, I.; GONZÁLEZ, R.; ONOFRE, J.; GARTZ-TONDORF, G. 2012. Sensibilidad y especificidad de la escala de Alvarado en el diagnóstico de apendicitis aguda comparada con TAC o ultrasonido en las primeras 24 horas de evolución. Rev. Cirujano General. 34(2):107-110.
6. DANE. 2005. Proyecciones de población total por años y sexo, según departamentos periodo 2005-2020. Cuadro 7: 50. Bogotá, Colombia.

7. DE QUESADA, L.; IVAL, M.; GONZÁLEZ, M. 2015. La escala de Alvarado como recurso clínico para el diagnóstico de la apendicitis aguda. Rev. Cubana de Cirugía.54:2.

8. HERNÁNDEZ, L.; DOMÍNGUEZ, D. 2012. Sensibilidad y especificidad de la escala de Alvarado en apendicitis aguda en el Hospital Regional de alta especialidad de Veracruz. Rev. Cirujano General. 34(3):179-184.

9. KHAN, I.; UR REHMAN, A. 2005. Application of Alvarado scoring system in diagnosis of acute appendicitis. J. Ayub. Med. Coll. Abbottabad. 17(3):41-44.

10. MINISTERIO DE SALUD. 1993. Resolución 8430 de 1993. Por la cual se establecen las normas científicas, técnicas y administrativas para la investigación en salud. Artículo. 11, sección a. Bogotá, Colombia.

11. OHLE, R.; O'REILLY, F.; O'BRIEN, K.; FAHEY, T.; DIMITROV, B. 2011. The Alvarado score for predicting acute appendicitis: A systematic review. BMC Medicine. 9(139):1-13.

12. OSPINA, J.; BARRERA, L.; MANRIQUE, F. 2011. Utilidad de una escala diagnóstica en casos de apendicitis aguda. Rev. Col. Cir. 26:234-241.

13. PAULSON, E.; KALADY, M.; PAPPAS, T. Suspected appendicitis. 2003. N. Engl. J. Med. 348(3):236-242.

14. RICCI, L.; FERREYRA, C.; CÓRDOBA, M.; RIOS, A.; STATTI, M. 2015. Apendicitis aguda según los criterios de Alvarado. Rev. Argent Cirug. 107(2):57-62.

15. ROSSINI, S.; HABERMAN, D.; GONZALEZ, R. 2009. Utilidad de la tomografía computada en pacientes con dolor en fosa iliaca derecha: Apendicitis aguda y su diagnóstico diferencial. Rev. Argent. Radiol. 73(1):51-63.

16. SANABRIA, A.; MORA, M.; DOMÍNGUEZ, L.; VEGA, V.; OSORIO, C. 2010. Validación de la escala diagnóstica de Alvarado en pacientes con dolor abdominal sugestivo de apendicitis en un centro de segundo nivel de complejidad. Rev. Col. Cir. 25(3):195-201. 
17. SANEI, B.; MAHMOODIEH, M.; HOSSEINPOUR, M. 2009. Evaluation of validity of Alvarado scoring system for diagnosis of acute appendicitis. Pak. J. Med. Sci. 25(2):298-301.

18. SINGH, K.; GUPTA, S.; PARGAL, P. 2008. Application of Alvarado scoring system in diagnosis of acute appendicitis. Jk Science. 10(2):84-86.

19. VEGA, C.; MUÑOZ, N.; CHIQUIZA, L.; BARRETO, S. 2013. Consenso cirujanos; COVE e infectología.
Guía de práctica clínica de apendicitis aguda, USS Tunal. Bogotá, Colombia.

20. VELÁZQUEZ, J.; GODÍNEZ, C.; VÁZQUEZ, M. 2010. Evaluación prospectiva de la escala de Alvarado en el diagnóstico de apendicitis aguda. Rev. Cirujano General. 32(1):17-23.

Recibido: Mayo 3 de 2016

Aceptado: Mayo 31 de 2017

Cómo citar:

Peralta R., K.V.; Caballero R., C.A.; Mora V., M. del P. 2017. Validez diagnóstica de la escala de Alvarado en pacientes con dolor abdominal sugestivo de apendicitis en un hospital de tercer nivel del sur de Bogotá. Rev. U.D.C.A Act. \& Div. Cient. 20(1): $5-11$. 